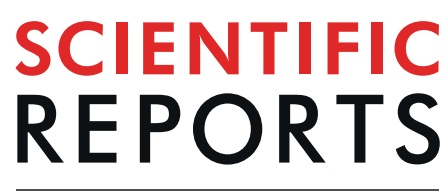

natureresearch

Check for updates

\title{
Maternal plasma metabolic markers of neonatal adiposity and associated maternal characteristics: The GUSTO study
}

\author{
Ai-Ru Chia ${ }^{1,2}$, Jamie V. de Seymour ${ }^{3}$, Gerard Wong ${ }^{4}$, Karolina Sulek ${ }^{5,6}$, Ting-Li Han ${ }^{5,7}$, \\ Elizabeth J. McKenzie ${ }^{5}$, Izzuddin M. Aris ${ }^{8}$, Keith M. Godfrey ${ }^{9}$, Fabian Yap ${ }^{10,11,12}$, \\ Kok Hian Tan ${ }^{13}$, Lynette Pei-Chi Shek ${ }^{4,14}$, Yung Seng Lee ${ }^{4,14,15}$, Michael S. Kramer ${ }^{1,16}$, \\ Neerja Karnani ${ }^{4}$, Mary Foong-Fong Chong ${ }^{2,4,18} \bowtie$ \& Philip N. Baker ${ }^{5,7,17,18}$
}

Infant adiposity may be related to later metabolic health. Maternal metabolite profiling reflects both genetic and environmental influences and allows elucidation of metabolic pathways associated with infant adiposity. In this multi-ethnic Asian cohort, we aimed to (i) identify maternal plasma metabolites associated with infant adiposity and other birth outcomes and (ii) investigate the maternal characteristics associated with those metabolites. In 940 mother-offspring pairs, we performed gas chromatography-mass spectrometry and identified 134 metabolites in maternal fasting plasma at 26-28 weeks of gestation. At birth, neonatal triceps and subscapular skinfold thicknesses were measured by trained research personnel, while weight and length measures were abstracted from delivery records. Gestational age was estimated from first-trimester dating ultrasound. Associations were assessed by multivariable linear regression, with $p$-values corrected using the Benjamini-Hochberg approach. At a false discovery rate of $5 \%$, we observed associations between 28 metabolites and neonatal sum of skinfold thicknesses ( 13 amino acid-related, 4 non-esterified fatty acids, 6 xenobiotics, and 5 unknown compounds). Few associations were observed with gestational duration, birth weight, or birth length. Maternal ethnicity, pre-pregnancy BMI, and diet quality during pregnancy had the strongest associations with the specific metabolome related to infant adiposity. Further studies are warranted to replicate our findings and to understand the underlying mechanisms.

\footnotetext{
${ }^{1}$ Department of Obstetrics \& Gynaecology, Yong Loo Lin School of Medicine, National University of Singapore, Singapore, Singapore. 'Saw Swee Hock School of Public Health, National University of Singapore, Singapore, Singapore. ${ }^{3}$ School of Sport, Exercise and Nutrition, Massey University, Auckland, New Zealand. ${ }^{4}$ Singapore Institute for Clinical Science, Agency for Science, Technology, and Research, Singapore, Singapore. ${ }^{5}$ Liggins Institute, University of Auckland, Auckland, New Zealand. ${ }^{6}$ Novo Nordisk Foundation Center for Protein Research, Faculty of Health and Medical Sciences, University of Copenhagen, Copenhagen, Denmark. ${ }^{7}$ First Affiliated Hospital of Chongqing Medical University, Chongqing, China. ${ }^{8}$ Division of Chronic Disease Research Across the Lifecourse, Department of Population Medicine Harvard Medical School and Harvard Pilgrim Health Care Institute, Boston, MA, USA. ${ }^{9}$ Medical Research Council Lifecourse Epidemiology Unit and National Institute for Health Research Southampton Biomedical Research Centre, University of Southampton and University Hospital Southampton National Health Service Foundation Trust, Southampton, United Kingdom. ${ }^{10}$ Duke-NUS Medical School, Singapore, Nanyang Technological University, Singapore, Singapore. ${ }^{11}$ Department of Pediatrics, KK Women's and Children's Hospital, Singapore, Singapore. ${ }^{12}$ Lee Kong Chian School of Medicine, Nanyang Technological University, Singapore, Singapore. ${ }^{13}$ Department of Reproductive Medicine, KK Women's and Children's Hospital, Singapore, Singapore. ${ }^{14}$ Department of Pediatrics, Yong Loo Lin School of Medicine, National University of Singapore, Singapore, Singapore. ${ }^{15}$ Khoo Teck Puat-National University Children's Medical Institute, National University Health System, Singapore, Singapore. ${ }^{16}$ Departments of Pediatrics and of Epidemiology, Biostatistics and Occupational Health, McGill University Faculty of Medicine, Montreal, Quebec, Canada. ${ }^{17}$ College of Life Sciences, University of Leicester, Leicester, UK. ${ }^{18}$ These authors jointly supervised this work: Mary Foong-Fong Chong and Philip N. Baker. ${ }^{\bowtie}$-mail: airu-chia@nus.edu.sg; mary_chong@nus.edu.sg
} 
Being born too early, too small, or too large is associated with impaired development and chronic diseases later in life ${ }^{1-3}$. Infants of similar weight often differ in adiposity ${ }^{4}$, and given the increasing trend of childhood obesity globally, investigation of infant adiposity is crucial in order to understand the early-life determinants of metabolic health $^{5}$.

Metabolomics is the study of low molecular weight metabolites $(<1000 \text { Daltons })^{6}$ downstream from the omics cascade (genomics, transcriptomics, and proteomics) and most closely reflects the metabolic phenotype ${ }^{7}$. Maternal metabolite profiling is of interest and value in perinatology, because it provides a snapshot of the maternal physiological status. It therefore reflects both genetic and lifestyle (environmental) influences, thus allowing elucidation of metabolic pathways associated with birth outcomes ${ }^{8-10}$. While studies among non-pregnant populations have reported variations in metabolomic profiles associated with ethnicity, diet, adiposity, and smoking status $^{11-14}$, it is unclear whether similar associations are observed during pregnancy, when the maternal body undergoes major physiological adaptions to support fetal development and growth ${ }^{15}$.

Several studies have demonstrated associations between maternal metabolite profiling during pregnancy and infant birth outcomes, based on biospecimens such as plasma ${ }^{16-22}$, urine ${ }^{22-26}$, amniotic fluid ${ }^{26-28}$, serum ${ }^{29-32}$, cervicovaginal fluid ${ }^{33-35}$, and hair ${ }^{36}$. Blood plasma is clinically accessible ${ }^{37}$ and potentially reflects processes in all organ systems. Compared to other biofluids, it is more comprehensive and contains the broadest array of metabolites ${ }^{6}$. Plasma metabolite profiles were typically analysed by liquid chromatography-mass spectrometry $(\mathrm{LC}-\mathrm{MS})^{16,17,19,21}$, flow injection analysis mass spectrometry (FIA-MS $)^{20}$, or nuclear magnetic resonance (NMR) $)^{18}$, but none by gas chromatography-mass spectrometry (GC-MS), which has been regarded as the gold standard for metabolite profiling of volatile, low molecular weight metabolites due to its greater chromatographic resolution ${ }^{8}$.

In addition, most previous studies were conducted in Western countries ${ }^{16-22}$ and had sample sizes under 100 participants ${ }^{17-19}$, with minimal or no adjusment for confounders ${ }^{16-18}$. Larger multi-ethnic studies are therefore warranted. In our multiethnic Asian mother-offspring cohort study, we aimed to (i) identify maternal plasma metabolites associated with gestational duration and offspring birth size and adiposity and (ii) investigate the maternal characteristics associated with those metabolites to examine the potential sources of metabolic variation.

\section{Methods}

Study participants. First-trimester pregnant women aged $18-50$ y were recruited from the National University Hospital and KK Women's and Children's Hospital between June 2009 and September 2010 to participate in the GUSTO (Growing Up in Singapore Towards healthy Outcomes) mother-offspring cohort ${ }^{38}$. Women were ineligible if their parents and spouses' parents had different ethnicity (i.e., Chinese, Malay, or Indian descent), received chemotherapy or psychotropic drugs, or had type 1 diabetes. This study was approved by the National Health Care Group Domain Specific Review Board (reference D/09/021) and the SingHealth Centralized Institutional Review Board (reference 2009/280/D). All research was performed in accordance with the relevant guidelines and informed consent was obtained from all participants upon recruitment.

Maternal metabolite assessment. Plasma sample preparation. We collected fasting blood samples at 26-28 week of gestation, processed within $4 \mathrm{~h}$, and stored at $-80^{\circ} \mathrm{C}$. Plasma samples were analysed in random order, and laboratory staff were blinded to birth outcomes. A $200 \mu \mathrm{L}$ aliquot of plasma was spiked with two internal standard mixes: $20 \mu \mathrm{L}$ of mix 1 prepared by dissolving $36 \mathrm{mg}$ citric acid- $\mathrm{d}_{4}, 34 \mathrm{mg}$ alanine- $\mathrm{d}_{4}, 26 \mathrm{mg}$ tryptophan- $\mathrm{d}_{5}, 23 \mathrm{mg}$ hexanoic- $\mathrm{d}_{11}$ acid, $21 \mathrm{mg}$ phenylalanine- $\mathrm{d}_{5}$, and $15 \mathrm{mg}$ tyrosine- $\mathrm{d}_{2}$ in $36 \mathrm{ml}$ of milli-Q water; and $20 \mu \mathrm{L}$ of mix 2 prepared by dissolving $29 \mathrm{mg}$ octanoic- $\mathrm{d}_{15}$ acid and $58 \mathrm{mg}$ stearic- $\mathrm{d}_{35}$ acid in $36 \mathrm{ml}$ of methanol. Quality control (QC) samples were prepared from a pool created from $40 \mu \mathrm{L}$ aliquots of all samples. The samples were dried using a SpeedVac Concentrator (Thermo Scientific) at $80 \mathrm{~Pa}$ with no heating and stored at $-80^{\circ} \mathrm{C}$.

Extraction of metabolites and derivatization (to increase compound volatility) were based on a published protocol $^{39}$. Dried samples were suspended in $500 \mu \mathrm{L}$ cold methanol-water solution ( $\left.50 \% \mathrm{vol} / \mathrm{vol}\right)$, vortexed, centrifuged at $3500 \mathrm{rpm}$ for $5 \mathrm{~min}$ at $-4^{\circ} \mathrm{C}$, and the supernatant was collected. The same extraction process was repeated for methanol-water $(80 \% \mathrm{vol} / \mathrm{vol})$ and methanol solutions. The pooled supernatants were dried using a SpeedVac Concentrator and stored at $-80^{\circ} \mathrm{C}$.

Dried samples were suspended in $400 \mu \mathrm{L}$ sodium hydroxide $(1 \mathrm{~mol} / \mathrm{L}), 334 \mu \mathrm{L}$ methanol and $68 \mu \mathrm{L}$ pyridine in a silanized glass tube to reduce compound adherence to the glass wall (Thermo Scientific). The derivatization was initiated by adding $40 \mu \mathrm{L}$ methyl chloroformate, followed by vigorous vortexing for $30 \mathrm{~s}$. This process was repeated before adding $400 \mu \mathrm{L}$ chloroform and $800 \mu \mathrm{L}$ sodium bicarbonate $(50 \mathrm{mmol} / \mathrm{L})$, vortexing for $10 \mathrm{~s}$ after each addition. Derivatised samples were centrifuged at $2000 \mathrm{rpm}$ for $3 \mathrm{~min}$; the aqueous layer was removed and dehydrated with $300 \mathrm{mg}$ anhydrous sodium sulphate before gas chromatography-mass spectrometry (GC-MS) analysis. Negative controls (without the addition of plasma and internal standards) underwent the same processing.

Gas chromatography-mass spectrometry (GC-MS) analysis. We analysed the samples on a GC-MS system-a GC7890B coupled to an MSD5977A operated at $70 \mathrm{eV}$ electron ionization (Agilent Technologies) - equipped with a ZB- 1701 column, $30 \mathrm{~m} \times 0.25 \mathrm{~mm} \times 0.15 \mu \mathrm{m}$ with $5 \mathrm{~m}$ guard column (Phenomenex), at a constant flow rate of $1.0 \mathrm{~mL} / \mathrm{min}$ of helium. Based on a published protocol ${ }^{39}$, samples $(1 \mu \mathrm{L})$ were injected under pulsed splitless mode (for trace analyses) with the inlet at $290^{\circ} \mathrm{C}(180 \mathrm{kPa}$ for $1 \mathrm{~min}, 50 \mathrm{~mL} / \mathrm{min}$ purge flow after $1 \mathrm{~min})$. The GC oven temperature was initially held at $45^{\circ} \mathrm{C}$ for $2 \mathrm{~min}$, after which it was raised to $180^{\circ} \mathrm{C}$ at $9{ }^{\circ} \mathrm{C} / \mathrm{min}$ and held for $5 \mathrm{~min}$. Subsequently, the temperature was raised to $220^{\circ} \mathrm{C}$ at $40^{\circ} \mathrm{C} / \mathrm{min}$ and held for $5 \mathrm{~min}$. The temperature was raised again to $240^{\circ} \mathrm{C}$ at $40^{\circ} \mathrm{C} / \mathrm{min}$ and held for $11.5 \mathrm{~min}$. Finally, the temperature was raised to $280^{\circ} \mathrm{C}$ at $40^{\circ} \mathrm{C} /$ min and held for $10 \mathrm{~min}$. The interface was kept at $250^{\circ} \mathrm{C}$ and the quadrupole at $130^{\circ} \mathrm{C}$. The detector operated in scan mode, started after $5.5 \mathrm{~min}$ with a mass range between $38-550$ atomic mass units at $2.9 \mathrm{scans} / \mathrm{s}$. Negative controls were injected at the beginning of each run and QCs were run every seventh sample. 
Data processing. The GC-MS data were deconvoluted and metabolites were identified by the Automated Mass Spectral Deconvolution and Identification system software ${ }^{40}$ with the in-house methyl chloroformate ${ }^{39}$ and publicly available National Institute of Standards and Technology 14 mass spectra library ${ }^{41}$. We used the R Metab package for peak integration ${ }^{42}$. Features present in $<5 \%$ of all samples and contaminants, identified by comparison with negative control samples, were excluded. Visual inspection of all extracted ion chromatograms was performed, and any integration errors were corrected. Each metabolite was normalised by one of the seven internal standards, chosen based on the highest linear correlation to the metabolite in the QC samples. All metabolites with an $\mathrm{R}^{2}<0.75$ with each and every internal standard were omitted from the analysis. We used median centering to align batches based on the correction factors derived from QCs in each of the batches ${ }^{43}$. Values below the limit of detection were assigned half of the minimum value for that metabolite ${ }^{44}$. We identified 134 metabolites; the median (IQR) coefficient of variation for all metabolites in the QC samples was 10.3 (8.2-13.4) and all were below the $30 \%$ threshold ${ }^{45}$. Metabolite values were log-transformed and centered before statistical analysis. Of the 134 metabolites, 43 were identified ( $80-100 \%$ match to a reference standard), 11 were putatively identified (80-100\% mass spectral match), 38 were tentatively identified (60-79\% mass spectral match), and 42 were unknown ( $<60 \%$ mass spectral match). We set the level of identification based on mass spectral matching levels and according to acceptable practices for chemical journals ${ }^{46}$.

Infant characteristics. Gestational age (GA) was estimated by a dating scan in the first trimester; preterm birth was defined as delivery of a live birth $<37$ weeks of gestation. Birth weight was measured shortly after birth to the nearest $1 \mathrm{~g}$ (SECA 334; SECA Corp.), and recumbent length measured to the nearest $0.5 \mathrm{~cm}$ from the top of the head to the soles of the feet (SECA 210). Sex-specific birth weight-for-GA z-scores were derived using a global birth weight reference ${ }^{47}$ adapted for the GUSTO population ${ }^{48}$. Triceps and subscapular skinfold thicknesses, which have greater discriminative power than other anthropometric measurements for neonatal total body adiposity ${ }^{49}$, were measured in triplicate to the nearest $0.2 \mathrm{~mm}$ on the right side of the body by anthropometrists and summed (Holtain Skinfold Caliper; Holtain Ltd.). All research personnel were trained according to standardized procedures obtained from the PhenX toolkit ${ }^{50}$. Infant sex was retrieved from birth delivery reports.

Maternal characteristics. Maternal age (in years, continuous), ethnicity (Chinese, Malay, or Indian), education (before secondary, postsecondary, or university), and self-reported pre-pregnancy weights were collected during recruitment. At 26-28 weeks' gestation, maternal weight was measured to the nearest $0.1 \mathrm{~kg}$ (SECA 803) and standing height was measured in duplicate to the nearest $0.1 \mathrm{~cm}$ from the top of the head to the heels (SECA 213). Pre-pregnancy underweight (BMI $<18.5 \mathrm{~kg} / \mathrm{m}^{2}$ or $\geq 18.5 \mathrm{~kg} / \mathrm{m}^{2}$ ), pre-pregnancy overweight (BMI $\geq 23 \mathrm{~kg} /$ $\mathrm{m}^{2}$ or $\left.<23 \mathrm{~kg} / \mathrm{m}^{2}\right)^{51}$ was determined and weight gain until $26-28$ weeks' gestation (in $\mathrm{kg}$, continuous) was calculated by subtracting pre-pregnancy weight from weight. Blood glucose levels were measured after fasting and two hours after a $75 \mathrm{~g}$ oral glucose load at 26-28 weeks' gestation. Women were diagnosed with gestational diabetes (yes or no) if fasting glucose was $\geq 7.0 \mathrm{mmol} / \mathrm{L}$ and/or 2 -hour post-glucose was $\geq 7.8 \mathrm{mmol} / \mathrm{L}$ according to the World Health Organization diagnostic criteria ${ }^{52}$. At $26-28$ weeks' gestation, information on diet quality during pregnancy (measured using the Healthy Eating Index for pregnant women in Singapore, continuous) ${ }^{53}$, physical activity (inactive, sufficiently active, or highly active) ${ }^{54}$, tobacco smoke exposure (plasma cotinine $<0.17 \mathrm{ng} / \mathrm{mL}$ and no environmental tobacco smoke exposure, $<0.17 \mathrm{ng} / \mathrm{mL}$ and self-reported environmental tobacco smoke exposure, $0.17-13.99 \mathrm{ng} / \mathrm{mL}$, or $\geq 14 \mathrm{ng} / \mathrm{mL})^{55}$, and alcohol consumption during pregnancy (yes or no) were also obtained. Parity (nulliparous or multiparous) and hypertensive disorders (yes or no) including chronic hypertension, pregnancy-induced hypertension, and pre-eclampsia were retrieved from delivery medical notes.

Statistical analysis. Among the 1152 spontaneously conceived singleton pregnancies, 940 mothers who provided their blood samples and had metabolite data and information on their offspring birth outcomes were included in the analysis (Supplemental Fig. 1).

Maternal and child characteristics were summarized according to quintiles of gestational age, birth weight, birth length, and sum of neonatal skinfold thicknesses. P values for trend were assessed by modelling the median value of the quintiles in linear regression for continuous variables or Cochran-Mantel-Haenszel tests for categorical variables.

The associations of 134 maternal metabolites with offspring birth outcomes (i.e. gestational duration, birth weight, birth length, and sum of skinfold thicknesses) were assessed by multiple linear regression using a separate model for each metabolite. We conducted sensitivity analysis on sex-specific birth weight-for-GA z-scores to examine the robustness of our study results. The models were adjusted for infant sex and maternal age, parity, education, ethnicity, pre-pregnancy BMI, weight gain until 26-28 weeks' gestation, height, tobacco smoke exposure, physical activity, diet quality, and gestational diabetes. The analysis approach is adopted and supported by recently published studies ${ }^{21,31}$. Metabolites associated with birth outcomes with a Benjamini-Hochberg false discovery rate (FDR) corrected q-value $<0.10^{56}$ were selected for the next step of analysis ${ }^{57}$.

For the second aim of the study, relationships between maternal characteristics and those selected metabolites were independently examined using multiple linear regression. Metabolite z-scores were modelled as dependent variables while the independent variables were maternal characteristics. To derive individual estimates for each ethnic group, we created three variables: Chinese (yes or no), Malay (yes or no), and Indian (yes or no) and fitted the model with one ethnic variable at a time while adjusting for other maternal characteristics.

Missing data (height $(n=9)$, diet quality $(n=9)$, physical activity $(n=10)$, education $(n=11)$, alcohol use $(n=25)$, gestational diabetes $(n=36)$, pre-pregnancy BMI $(n=66)$, weight gain until 26-28 weeks of gestation $(n=73)$, and prenatal tobacco smoke exposure $(n=73)$ ) were estimated by 20 imputations by the Markov chain Monte Carlo method; the reported results are those from the pooled analysis. To evaluate whether the imputation 
of missing data may have affected the results, we carried out a sensitivity analysis on participants with no missing data $(n=722)$.

We investigated potential effect modification by ethnicity by including an interaction term (ethnicity $\mathrm{x}$ metabolite) in the regression models. Ethnic differences were further explored by stratified analyses if the interaction term was significant (q-value $<0.05$ ). We performed sensitivity analyses to restrict analysis to term births (except for the analysis of gestational age). We also limited the analysis to women without hypertensive disorders, pre-eclampsia, or gestational diabetes $(n=536)$ in a separate sensitivity analysis.

All statistical analysis was performed in Stata 14 (StataCorp LP, USA). We considered q-values $<0.05$ statistically significant and $0.05<\mathrm{q}$-values $<0.10$ as trends.

Ethics approval and consent to participate. This study was approved by the National Health Care Group Domain Specific Review Board (reference D/09/021) and the SingHealth Centralized Institutional Review Board (reference 2009/280/D). All participants gave informed consent upon recruitment.

\section{Results}

Characteristics of participants and birth outcomes. This cohort comprised 55\% Chinese, 26\% Malays, and 19\% Indians. The mean and standard deviation of maternal age was $30.5 \pm 5.1 \mathrm{y}$, and $43 \%$ were overweight before pregnancy. Among the 940 infants, 7.6\% were born preterm; the means and standard deviations of birth weight, birth length, and sums of neonatal skinfold thicknesses were $3094 \pm 451 \mathrm{~g}, 48.6 \pm 2.3 \mathrm{~cm}$, and $10.3 \pm 2.2 \mathrm{~mm}$, respectively. Women with shorter gestational duration were more likely to have lower education, greater exposure to tobacco smoke during pregnancy, and hypertensive disorders. Heavier and longer infants were more likely to be born to mothers who were older, taller, had higher education, and had lower exposure to tobacco smoke. Infants with a lower sum of skinfold thicknesses were more likely to be born to mothers who were nulliparous and had higher education and higher diet quality. Mothers who were overweight before pregnancy and those of higher weight gain up to 26-28 weeks' gestation were more likely to have infants who were heavier and have higher sum of skinfolds. Chinese infants were generally longer than Malay infants. Only results from the extreme quintiles are presented in Table 1 while all results are presented in Supplemental Table S1.

Associations of maternal metabolites and birth outcomes. Only metabolites associated with birth outcomes (q-value $<0.05$ ) are presented in Table 2, while all results are presented in Supplemental Table S2. One metabolite was found to be inversely associated with gestational duration, and another metabolite with birth weight; they were tentatively identified as pyrazole, 3-nitro- and 10-nonadecenoic acid, methyl ester respectively. There were trends toward association of four metabolites and birth length, of which two were related to branched chain amino acids (3-methyl-2-oxopentanoic acid and isoleucine).

We observed inverse associations between 28 metabolites and infants' sum of skinfold thicknesses at birth -13 amino acid-related, 4 fatty acids, 6 xenobiotics, and 5 unknown compounds. Branched-chain fatty acids, medium-chain fatty acids (C10:0 and C12:0), and omega- 6 fatty acids (C20:2 and C22:4) showed inverse trends with sum of skinfolds while 2-hydroxybutyric acid and 2-hydroisobutyric acid showed positive trends.

Potential interactions between ethnicity and metabolite associations for birth weight (5 metabolites) and birth length (8 metabolites) but not gestational age or sum of skinfolds (Supplemental Table S3). Stratified analyses were conducted, and higher branched-chain fatty acids were associated with higher birth weight and longer birth length in Indians but not in Chinese and Malays.

Associations of maternal characteristics and infant adiposity-related metabolites. Figure 1 summarizes the relationships between maternal characteristics and the infant adiposity-related metabolites. All results and estimates are presented in Supplemental Table S4. Ethnicity had the highest number of significant and independent associations with metabolites related to infant adiposity $(n=26)$. Generally, Chinese and Indian women showed contrasting associations. For example, levels of branched chain amino acids and 2-hydroxybutyric acid were higher in Chinese women but lower in Indian women. Metabolite profiles of Chinese women shared similarities with those of women who were overweight before pregnancy ( $n=16$ metabolites showed concordant direction of effect estimates).

Among the modifiable factors, pre-pregnancy overweight and maternal diet quality had the strongest associations with the metabolome $(n=17$ and $n=15$ metabolites, respectively). Few significant associations were found with parity, maternal age, tobacco smoke exposure during pregnancy, and gestational diabetes, and none with pre-pregnancy underweight, physical activity, and alcohol consumption during pregnancy.

Branched chain fatty acids were associated with most maternal factors. Lower levels were observed in participants who were exposed to tobacco smoke during pregnancy, multiparous, overweight, or of Chinese ethnicity, but higher levels were seen in Malay and Indian women and women with better diet quality.

Sensitivity analysis. Results were largely consistent when we examined sex-specific birth weight-for-GA $\mathrm{Z}$-scores or adjusted for fasting or postprandial plasma glucose level instead of gestational diabetes. When we restricted our analysis of sum of skinfold thicknesses to participants with no missing data $(n=722)$, term births, or women without hypertensive disorders, or gestational diabetes $(n=536)$, results remained similar (data not shown). 


\begin{tabular}{|c|c|c|c|c|c|c|c|c|c|c|c|c|}
\hline \multirow[b]{3}{*}{ Characteristics } & \multicolumn{3}{|c|}{ Gestational duration (week) } & \multicolumn{3}{|c|}{ Birth weight (g) } & \multicolumn{3}{|c|}{ Birth length $(\mathrm{cm})$} & \multicolumn{3}{|c|}{$\begin{array}{l}\text { Sum of triceps and subscapular } \\
\text { skinfold (mm) }\end{array}$} \\
\hline & \multirow{2}{*}{$\begin{array}{l}\text { Q1 } \\
36(37-38)\end{array}$} & \multirow{2}{*}{\begin{tabular}{|l} 
Q5 \\
$40(40-40)$
\end{tabular}} & \multirow[b]{2}{*}{ P-trend } & \multirow{2}{*}{\begin{tabular}{|l|} 
Q1 \\
2580 \\
$(2383-$ \\
$2675)$
\end{tabular}} & \multirow{2}{*}{\begin{tabular}{|l|} 
Q5 \\
3640 \\
$(3550-$ \\
$3805)$
\end{tabular}} & \multirow[b]{2}{*}{ P-trend } & \multirow{2}{*}{\begin{tabular}{|l|} 
Q1 \\
$46(46-47)$ \\
\end{tabular}} & \multirow{2}{*}{\begin{tabular}{|l|} 
Q5 \\
$52(52-53)$
\end{tabular}} & \multirow[b]{2}{*}{ P-trend } & \multirow{2}{*}{\begin{tabular}{|l|} 
Q1 \\
7.8 \\
$(7.3-8.2)$
\end{tabular}} & \multirow{2}{*}{\begin{tabular}{|l|} 
Q5 \\
$13.4(12.6-$ \\
$14.8)$
\end{tabular}} & \multirow[b]{2}{*}{ P-trenc } \\
\hline & & & & & & & & & & & & \\
\hline Maternal age, $y$ & $30.3 \pm 5.3$ & $30.4 \pm 4.9$ & 0.84 & $29.9 \pm 5.4$ & $31.2 \pm 4.5$ & 0.01 & $30.0 \pm 5.4$ & $31.6 \pm 4.3$ & 0.002 & $29.9 \pm 4.9$ & $31.3 \pm 5.1$ & 0.02 \\
\hline $\begin{array}{l}\text { Weight gain until 26-28 } \\
\text { weeks, kg }\end{array}$ & $9.2 \pm 4.3$ & $8.9 \pm 4.6$ & 0.64 & $7.9 \pm 4.1$ & $10.2 \pm 4.7$ & $<0.001$ & $8.5 \pm 4.9$ & $8.8 \pm 3.8$ & 0.04 & $8.7 \pm 4.6$ & $9.9 \pm 4.7$ & 0.01 \\
\hline Height, $\mathrm{cm}$ & $158 \pm 5.8$ & $159 \pm 5.6$ & 0.24 & $157 \pm 5.4$ & $159 \pm 5.6$ & 0.002 & $157 \pm 5.7$ & $159 \pm 5.9$ & $<0.001$ & $159 \pm 5.5$ & $158 \pm 5.5$ & 0.08 \\
\hline HEI-SGP score & $51 \pm 13$ & $53 \pm 14$ & 0.10 & $51 \pm 13$ & $52 \pm 15$ & 0.84 & $50 \pm 12$ & $57 \pm 13$ & $<0.001$ & $54 \pm 14$ & $49 \pm 13$ & $<0.001$ \\
\hline Ethnicity, \% & & & 0.23 & & & 0.23 & & & 0.05 & & & 0.54 \\
\hline Chinese & 46 & 58 & & 55 & 57 & & 48 & 63 & & 54 & 56 & \\
\hline Malay & 35 & 20 & & 23 & 27 & & 33 & 16 & & 27 & 32 & \\
\hline Indian & 19 & 22 & & 22 & 17 & & 18 & 20 & & 19 & 12 & \\
\hline Education, \% & & & 0.04 & & & 0.02 & & & $<0.001$ & & & 0.005 \\
\hline None/primary/secondary & 35 & 29 & & 37 & 31 & & 37 & 16 & & 29 & 37 & \\
\hline Postsecondary & 38 & 27 & & 37 & 33 & & 39 & 28 & & 32 & 35 & \\
\hline University & 28 & 44 & & 26 & 36 & & 24 & 56 & & 39 & 28 & \\
\hline Physical activity, \% & & & 0.45 & & & 0.90 & & & 0.78 & & & 0.005 \\
\hline Inactive & 27 & 31 & & 32 & 31 & & 30 & 26 & & 38 & 28 & \\
\hline Sufficiently active & 52 & 52 & & 50 & 48 & & 52 & 50 & & 50 & 52 & \\
\hline Highly active & 20 & 17 & & 18 & 20 & & 18 & 25 & & 12 & 21 & \\
\hline Plasma cotinine, $\%$ & & & $<0.001$ & & & 0.002 & & & $<0.001$ & & & 0.65 \\
\hline $\begin{array}{l}<0.17 \mathrm{ng} / \mathrm{mL} \& \text { no ETS } \\
\text { exposure }\end{array}$ & 43 & 68 & & 45 & 58 & & 41 & 70 & & 51 & 48 & \\
\hline $\begin{array}{l}<0.17 \mathrm{ng} / \mathrm{mL} \& \text { self- } \\
\text { reported ETS }\end{array}$ & 31 & 19 & & 36 & 30 & & 35 & 21 & & 27 & 34 & \\
\hline $0.17-13.99 \mathrm{ng} / \mathrm{mL}$ & 19 & 10 & & 12 & 11 & & 18 & 9.4 & & 15 & 18 & \\
\hline$\geq 14 \mathrm{ng} / \mathrm{mL}$ & 7.0 & 2.3 & & 7.9 & 1.7 & & 7.0 & 0.0 & & 7.9 & 0.6 & \\
\hline Pre-pregnancy overweight, \% & 44 & 42 & 0.89 & 37 & 50 & 0.002 & 44 & 45 & 0.92 & 37 & 57 & 0.003 \\
\hline Nulliparous, \% & 42 & 51 & 0.06 & 44 & 36 & 0.17 & 41 & 46 & 0.79 & 51 & 32 & $<0.001$ \\
\hline $\begin{array}{l}\text { Alcohol use during } \\
\text { pregnancy, \% }\end{array}$ & 2.9 & 1.1 & 0.11 & 2.2 & 0.6 & 0.10 & 2.7 & 1.1 & 0.24 & 2.7 & 1.2 & 0.35 \\
\hline Gestational diabetes, $\%$ & 23 & 17 & 0.13 & 19 & 23 & 0.80 & 17 & 28 & 0.06 & 14 & 22 & 0.43 \\
\hline Hypertensive disorders, $\%$ & 8.1 & 1.6 & $<0.001$ & 5.3 & 3.7 & 0.30 & 4.9 & 1.0 & 0.05 & 3.3 & 7.1 & 0.15 \\
\hline Male infant, $\%$ & 58 & 52 & 0.26 & 48 & 64 & $<0.001$ & 45 & 66 & $<0.001$ & 60 & 37 & $<0.001$ \\
\hline
\end{tabular}

Table 1. Characteristics of participants according to quintiles of birth outcomes. Only extreme quintiles are reported while all results are presented in Supplemental Table S1. Values are means \pm SDs or medians (IQR). P-trends were assessed by modelling the median value of the quintiles in the linear regression analysis for continuous variables or Cochran-Mantel-Haenszel tests for categorical variables. There were missing data for pre-pregnancy BMI $(n=66)$, weight gain until 26-28 weeks $(n=73)$, height $(n=9)$, healthy eating index $(n=9)$, education $(n=11)$, physical activity $(n=10)$, plasma cotinine $(n=73)$, alcohol $(n=25)$, and gestational diabetes $(n=36)$. ETS, environmental tobacco smoke; HEI-SGP, Healthy Eating Index for pregnant women in Singapore; NA, not applicable; Q, quintile.

\section{Discussion}

In this multi-ethnic Asian cohort of 940 mother-offspring pairs, maternal levels of amino acid-related metabolites, non-esterified fatty acids, and xenobiotics were associated with neonatal sum of triceps and subscapular skinfold thicknesses. Few associations were observed with gestational duration, birth weight, or birth length. Maternal ethnicity, pre-pregnancy BMI, and diet quality during pregnancy had the strongest associations with the specific metabolome related to infant adiposity.

We are aware of one study that has examined the association between the maternal plasma metabolome and infant adiposity at birth ${ }^{21}$. In that United States study $(n=121)$, alkyl-linked phosphatidylcholines containing fatty acid 20:4 in the third trimester were associated with lower newborn body fat. However, differences in the analytical approach (targeted versus untargeted) and analytical platform (LC-MS versus GC-MS) limit our ability to compare our results to those of the previous study.

We observed an inverse association between amino acid-related metabolites and infant skinfold thickness. This finding is in line with our previous work, which showed higher maternal protein intake associated with lower abdominal internal adiposity in neonate ${ }^{58}$. However, we cautioned that protein intake does not necessarily mirror plasma concentrations of amino acids ${ }^{59,60}$ and that metabolite levels are also influenced by other maternal factors (e.g., ethnicity and pre-pregnancy overweight; see Fig. 1). 


\begin{tabular}{|c|c|c|c|c|c|}
\hline Metabolites & Metabolic sub-pathway & CAS number & $\begin{array}{l}\text { Match } \\
(\%)\end{array}$ & $\beta(95 \% \mathrm{CI})^{1}$ & q-value \\
\hline \multicolumn{6}{|l|}{ Duration of gestation, weeks } \\
\hline Pyrazole, 3-nitro- & Chemical & $26621-44-3$ & 76 & $-0.81(-1.24,-0.38)$ & 0.03 \\
\hline \multicolumn{6}{|l|}{ Birth weight, $\mathrm{g}$} \\
\hline 10-Nonadecenoic acid, methyl ester (C19:1) & $\begin{array}{l}\text { Monounsaturated Fatty acid; } \\
\text { Chemical }\end{array}$ & 56599-83-8 & 66 & $-190(-293,-88.0)$ & 0.04 \\
\hline \multicolumn{6}{|c|}{ Sum of triceps and subscapular skinfold thicknesses, $\mathbf{m m}$} \\
\hline \multicolumn{6}{|l|}{ Amino acid-related } \\
\hline Asparagine & Alanine \& Aspartate & $70-47-3$ & 81 & $-1.17(-1.74,-0.59)$ & $<0.001$ \\
\hline Creatinine & Creatine & $60-27-5$ & 93 & $-0.68(-1.05,-0.30)$ & 0.01 \\
\hline Methionine & $\begin{array}{l}\text { Cysteine, Methionine, SAM, } \\
\text { Taurine }\end{array}$ & $63-68-3$ & 97 & $-1.05(-1.54,-0.55)$ & $<0.001$ \\
\hline Glutamine & Glutamate & $56-85-9$ & 81 & $-0.82(-1.28,-0.36)$ & 0.01 \\
\hline Glycine & Glycine, Serine, Threonine & $56-40-6$ & 87 & $-0.80(-1.30,-0.31)$ & 0.01 \\
\hline Serine & Glycine, Serine, Threonine & $56-45-1$ & 76 & $-1.14(-1.71,-0.57)$ & $<0.001$ \\
\hline Threonine & Glycine, Serine, Threonine & $72-19-5$ & 93 & $-0.82(-1.30,-0.34)$ & 0.01 \\
\hline Lysine & Lysine & $56-87-1$ & 92 & $-0.91(-1.49,-0.33)$ & 0.02 \\
\hline Phenylalanine & Phenylalanine \& Tyrosine & $63-91-2$ & 95 & $-1.10(-1.73,-0.46)$ & 0.01 \\
\hline Tyrosine & Phenylalanine \& Tyrosine & $60-18-4$ & 92 & $-1.08(-1.59,-0.57)$ & $<0.001$ \\
\hline Ornithine & $\begin{array}{l}\text { Urea Cycle; Arginine \& } \\
\text { Proline }\end{array}$ & $70-26-8$ & 92 & $-0.55(-0.94,-0.15)$ & 0.03 \\
\hline Isoleucine & Valine, Leucine, Isoleucine & $73-32-5$ & 99 & $-0.68(-1.05,-0.30)$ & 0.01 \\
\hline Leucine & Valine, Leucine, Isoleucine & $61-90-5$ & 100 & $-0.73(-1.13,-0.34)$ & 0.01 \\
\hline \multicolumn{6}{|l|}{ Lipids } \\
\hline 2-Methyloctadecanoic acid & $\begin{array}{l}\text { Branched Chain Fatty Acid; } \\
\text { Chemical }\end{array}$ & $7217-83-6$ & 64 & $-0.73(-1.22,-0.25)$ & 0.02 \\
\hline Hexadecanoic acid, 14-methyl-, methyl ester & $\begin{array}{l}\text { Branched Chain Fatty Acid; } \\
\text { Chemical }\end{array}$ & $2490-49-5$ & 82 & $-0.46(-0.80,-0.13)$ & 0.03 \\
\hline Dodecanoic acid (C12:0) & Medium Chain Fatty Acid & $143-07-7$ & 94 & $-0.36(-0.59,-0.13)$ & 0.02 \\
\hline $\begin{array}{l}\text { trans-Vaccenic acid OR Oleic acid OR cis-Vaccenic } \\
\text { acid (C18:1) }\end{array}$ & Monounsaturated Fatty acid & $693-72-1$ & 79 & $-1.18(-1.98,-0.39)$ & 0.02 \\
\hline \multicolumn{6}{|l|}{ Xenobiotics } \\
\hline 2-Hydroxyisobutyric acid & Chemical & 594-61-6 & 67 & $0.81(0.19,1.44)$ & 0.05 \\
\hline 5-Aminoimidazole-4-carboxylic acid, methyl ester & Chemical & 4919-00-0 & 81 & $-1.13(-1.79,-0.47)$ & 0.01 \\
\hline Ethyl 2,5,8,11-tetraoxatridecan-13-oate & Chemical & $91719382^{*}$ & 73 & $-0.75(-1.26,-0.24)$ & 0.02 \\
\hline $\begin{array}{l}\text { glycine, N,N-bis(2-methoxy-2-oxoethyl)-, methyl } \\
\text { ester }\end{array}$ & Chemical & $22241-07-2$ & 83 & $0.27(0.18,0.35)$ & $<0.001$ \\
\hline $\begin{array}{l}\text { 1-Leucine, N-methoxycarbonyl-, ethyl ester OR } \\
\text { 2,2,2-trifluoroethyl ester }\end{array}$ & Chemical & 88406-43-3 & 90 & $-0.41(-0.71,-0.11)$ & 0.03 \\
\hline \multicolumn{6}{|l|}{ Unknown compounds } \\
\hline $\begin{array}{l}\text { 5-Cyano-4-methoxyamino-7-phenyl-hept-6-enoic } \\
\text { acid, methyl ester }\end{array}$ & - & - & 48 & $-0.81(-1.32,-0.30)$ & 0.02 \\
\hline 5-Octadecenoic acid, methyl ester & - & - & 58 & $-1.31(-2.24,-0.38)$ & 0.03 \\
\hline 7-Benzofuranamine, 2-methyl- & - & - & 49 & $-0.80(-1.29,-0.32)$ & 0.01 \\
\hline Benzene, hexyl- & - & - & 59 & $-1.15(-1.79,-0.50)$ & 0.01 \\
\hline $\begin{array}{l}\text { Imidazo[4,5-e][1,4]diazepin-8 }(1 \mathrm{H}) \text {-one, } \\
\text { 4,5,6,7-tetrahydro-4,7-dimethyl-5-thioxo- }\end{array}$ & - & - & 44 & $-0.74(-1.27,-0.20)$ & 0.04 \\
\hline Methylenedioxyamphetamine acetate & - & - & 57 & $-0.91(-1.47,-0.35)$ & 0.01 \\
\hline
\end{tabular}

Table 2. Associations between maternal plasma metabolite levels and infant birth outcomes (q-value $<0.05$ ). $\beta$ values are linear regression coefficients per SD increase in metabolite levels, adjusted for infant sex and maternal age, parity, education, ethnicity, pre-pregnancy BMI, weight gain until 26-28 weeks of gestation, height, physical activity, diet quality, plasma cotinine, and gestational diabetes. CAS, chemical abstracts service; SAM, s-adenosyl methionine. *PubChem CID.

Congruent with previous studies, we showed positive trends between maternal 2-hydroxybutyrate levels and infant skinfold thickness. 2-hydroxybutyrate is a known early biomarker of insulin resistance ${ }^{61}$ and maternal glucose intolerance has also been shown (in our cohort and others) to be strongly associated with higher skinfold thickness in infancy ${ }^{62,63}$.

Accumulating evidence shows that elevated fatty acid concentrations are not necessarily associated with increased adiposity and insulin resistance ${ }^{64}$. Some fatty acids play a vital role in fetal growth and development ${ }^{65}$. In particular, medium chain fatty acids, which were associated with lower infant skinfold thickness in our study, have been shown to be related to higher oxidative metabolism and reduced adiposity in both animal and human studies ${ }^{66}$. 


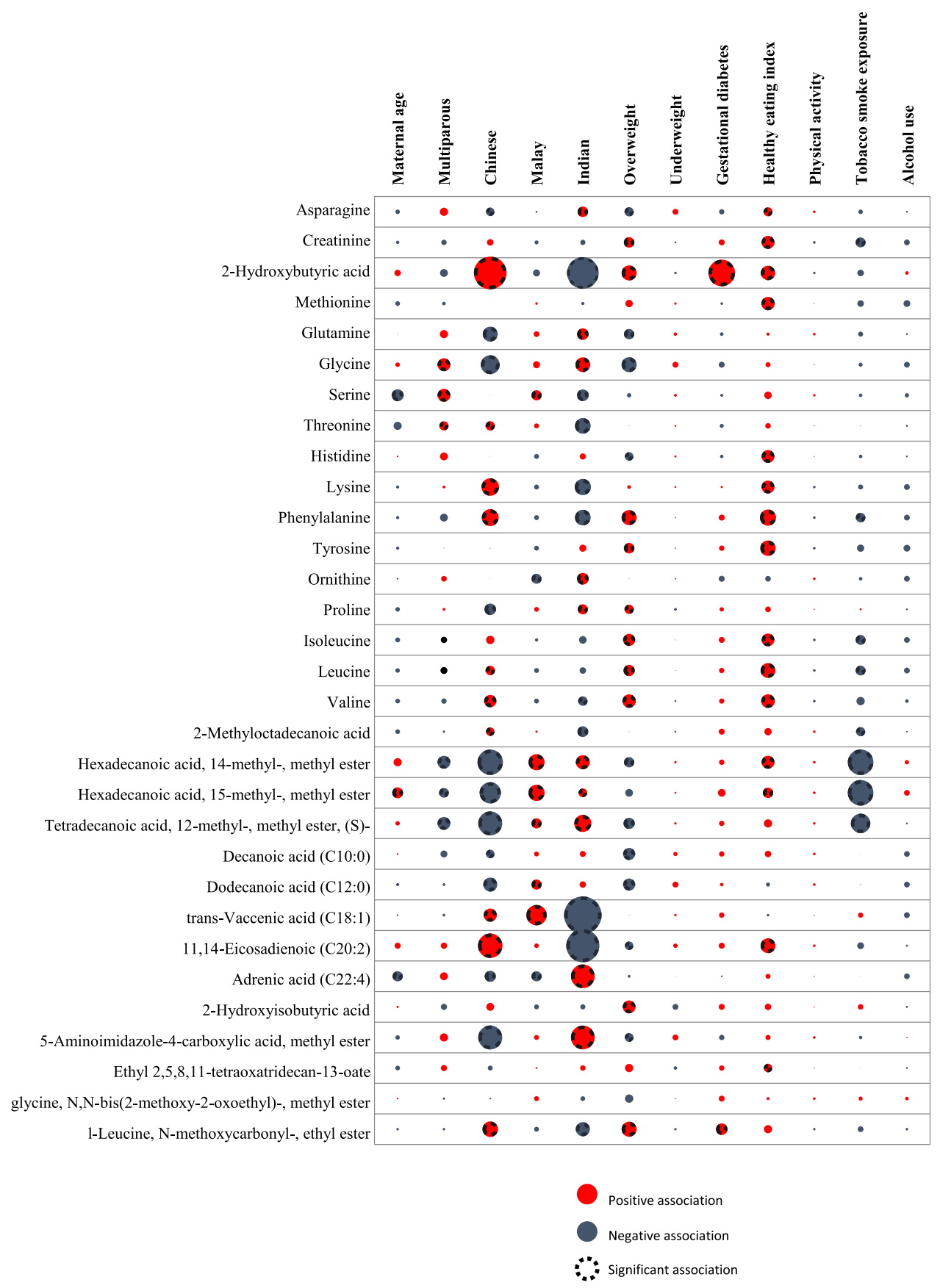

Figure 1. Maternal characteristics and infant adiposity-related metabolites. Multiple linear regression was performed with metabolite $\mathrm{z}$-scores modelled as dependent variables and maternal characteristics as independent variables. Each circle denotes negative log-transformed q-values derived by the BenjaminiHochberg procedure. Larger circles represent a lower q-value (i.e., a lower false discovery rate). (description for 'Positive association, 'Negative association' and 'Significant association').

Branched-chain fatty acids (BCFA) showed inverse trends with infant skinfold thickness. We also observed lower levels of BCFA in women exposed to tobacco smoke during pregnancy, and in women who were multiparous, overweight, or of Chinese ethnicity, while higher levels were observed in Malay and Indian women and in women with better diet quality (Fig. 1). BCFA can be synthesized de novo or obtained from ruminant fats and milk ${ }^{67}$. Recent studies have reported that BCFA levels are positively associated with insulin sensitivity and are lower in obese than in lean persons $s^{68,69}$. However, inadequate information on lipid metabolism during pregnancy limits our understanding of the underlying mechanisms.

Several xenobiotics were significantly associated with the sum of skinfolds. However, their precise function and origin are largely unknown, except for 2-hydroxyisobutyric acid (2-HIBA). 2-HIBA is a gut microbiota-associated metabolite ${ }^{70}$ and may also reflect environmental exposure of the fuel additive, methyl tert-butyl ether ${ }^{71}$. Higher 
levels of 2-HIBA, which was associated with higher infant skinfold thickness in our study, have been observed in the urine of obese people $\mathrm{e}^{72}$ and identified as a metabolic signature of adiposity $\mathrm{y}^{73}$ and diabetes mellitus $\mathrm{s}^{74}$.

We observed trends toward higher levels of branched-chain amino acid-related metabolites and longer birth length. This finding is in line with a cohort in United States examining cord blood metabolite patterns ${ }^{75}$. Pyrazole, 3-nitro- was found to be inversely associated with gestational duration, and 10-Nonadecenoic acid, methyl ester with birth weight. Based on their tentative identities, these metabolites have not been reported in earlier studies $^{16-19}$, likely due to differences in analytical techniques (GC-MS versus LC-MS or NMR). We found associations between higher branched chain fatty acids and higher birth weight and longer birth length in Indians, but this was not observed in Chinese and Malays. Ethnic-specific associations have not been reported in previous studies.

Maternal ethnicity had the strongest associations with the specific metabolome related to infant adiposity. Chinese and Indian women often showed contrasting associations; these ethnic differences were also observed in our earlier work in which Chinese infants, compared to Indians, were more susceptible to excessive neonatal adiposity from high maternal glycemia ${ }^{63}$. We also observed that metabolite profiles of Chinese women were similar to women who were overweight even though Chinese women had a lower mean BMI than Malay and Indians in our study (mean \pm SD: $21.6 \pm 3.4$ in Chinese; $24.2 \pm 5.4$ in Malay, $23.9 \pm 4.5$ in Indian). This may explain why the impact of increasing BMI on insulin resistance, C-reactive protein, and adiponectin levels is more pronounced in Chinese than in the other ethnic groups in Singapore ${ }^{76}$.

Among the modifiable factors, pre-pregnancy overweight and maternal diet quality were strongly associated with the metabolic signature of infant adiposity. Metabolite profiles have been shown to be reflective of diet quality ${ }^{13}$, but we did not observe similar diet-metabolite associations in our study. This difference may be attributable to differences in study population (pregnant versus non-pregnant) ${ }^{13}$ or in socio-cultural dietary habits. Congruent with our findings, pre-pregnancy BMI has been identified as an important driver of metabolic variation in a United States cohort ${ }^{16}$, and metabolites we found to be associated with overweight status have also been reported in other cohorts ${ }^{14,66,68,77-79}$. If our findings are replicated in other cohorts, formal mediation analysis would help sort out the degree to which the associations shown in Table 1 are mediated by the maternal metabolites or through other causal pathways

Strengths of our study include its prospective design (which minimizes interviewer and recall bias), the metabolomic profiling of a large multi-ethnic Asian population, the collection of lifestyle and clinical data (which permit adjustment for confounding factors), and the assessment of infant skinfold thicknesses, in addition to more common measures such as birth weight, which has been strongly associated with infant adipose stores and highlighted as a strong predictor of later child obesity risk ${ }^{16}$.

Several limitations of our study are worth noting. First, we have explored only the maternal plasma metabolome and concentrated on GC-MS analysis with methyl chloroformate derivatization. Although this method has optimized the detection of compounds with amino and/or carboxyl groups (which includes most metabolites of central carbon metabolism and key intermediates of cell metabolism ${ }^{39}$ ), our work could be complemented by employing different analytical techniques such as a variation of sample extraction methods or the use of multiple analytical platforms to obtain a more comprehensive overview of the metabolome. Second, we assessed maternal metabolites at 26-28 weeks of gestation, which precludes analysis of changes in metabolite levels across pregnancy. Third, we did not examine fetal growth throughout pregnancy; as a consequence, the temporal sequence between the maternal metabolome and infant birth size is unclear. Maternal metabolite profiles could have bi-directional relationships with fetal growth, such that abnormal fetal growth (e.g., intrauterine growth restriction) could influence maternal metabolite levels. Future studies should examine serial fetal biometry to address this issue. Last, as in any observational study, our findings could still be influenced by residual confounding such as imprecise measurements or underlying medical conditions despite the many potential confounding factors we adjusted for, and causality cannot be inferred.

In conclusion, we observed links between amino acid-related metabolites, non-esterified fatty acids, and infant adiposity at birth measured by sum of skinfold thickness. Few associations were observed with gestational duration, birth weight, or birth length. Maternal ethnicity, pre-pregnancy BMI, and diet quality had the strongest associations with the metabolome. Further independent studies are warranted to replicate our findings and to understand the underlying mechanisms.

\section{Data availability}

Data are available upon request because data request for analysis must be assessed and approved by the executive committee of the GUSTO study and administered by the main GUSTO study team of data management, which is a third-party source. Any matter related to data request, please contact Mary Chong Foong Fong at mary_chong@ nus.edu.sg

Received: 20 December 2019; Accepted: 12 May 2020;

Published online: 10 June 2020

\section{References}

1. Lee, A. C. C. et al. National and regional estimates of term and preterm babies born small for gestational age in 138 low-income and middle-income countries in 2010. Lancet Glob Health 1, e26-e36, https://doi.org/10.1016/S2214-109X(13)70006-8 (2013).

2. Henriksen, T. The macrosomic fetus: A challenge in current obstetrics. Acta Obstet. Gynecol. Scand. 87, 134-145, https://doi. org/10.1080/00016340801899289 (2008).

3. Chawanpaiboon, S. et al. Global, regional, and national estimates of levels of preterm birth in 2014: A systematic review and modelling analysis. Lancet Glob Health https://doi.org/10.1016/s2214-109x(18)30451-0 (2018).

4. Anand, S. S. et al. What accounts for ethnic differences in newborn skinfold thickness comparing south asians and white caucasians? Findings from the start and family birth cohorts. Int. J. Obes. (Lond.) 40, 239-244, https://doi.org/10.1038/ijo.2015.171 (2016). 
5. Biro, F. M. \& Wien, M. Childhood obesity and adult morbidities. Am. J. Clin. Nutr. 91, 1499S-1505S, https://doi.org/10.3945/ ajen.2010.28701B (2010).

6. Villas-Boas, S. G., Nielsen, J., Smedsgaard, J., Hansen, M. A. \& Roessner-Tunali, U. Metabolome analysis: An introduction. Vol. 24 (John Wiley \& Sons, 2007).

7. Horgan, R. P. \& Kenny, L. C. 'Omic' technologies: Genomics, transcriptomics, proteomics and metabolomics. Obstet. Gynecol. 13, 189-195, https://doi.org/10.1576/toag.13.3.189.27672 (2011).

8. Dunn, W. B. \& Ellis, D. I. Metabolomics: Current analytical platforms and methodologies. Trends Anal. Chem. 24, 285-294, https:// doi.org/10.1016/j.trac.2004.11.021 (2005).

9. Holmes, E., Wilson, I. D. \& Nicholson, J. K. Metabolic phenotyping in health and disease. Cell 134, 714-717, https://doi. org/10.1016/j.cell.2008.08.026 (2008).

10. Fanos, V., Atzori, L., Makarenko, K., Melis, G. B. \& Ferrazzi, E. Metabolomics application in maternal-fetal medicine. BioMed Res. Int. 2013 (2013).

11. Gu, F. et al. Cigarette smoking behaviour and blood metabolomics. Int. J. Epidemiol. 45, 1421-1432, https://doi.org/10.1093/ije/ dyv330 (2016).

12. van Valkengoed, I. G. M. et al. Ethnic differences in metabolite signatures and type 2 diabetes: A nested case-control analysis among people of south asian, african and european origin. Nutr. Diabetes 7, 300, https://doi.org/10.1038/s41387-017-0003-z (2017).

13. Akbaraly, T. et al. Association of circulating metabolites with healthy diet and risk of cardiovascular disease: Analysis of two cohort studies. Sci. Rep. 8, 8620, https://doi.org/10.1038/s41598-018-26441-1 (2018).

14. Würtz, P. et al. Metabolic signatures of adiposity in young adults: Mendelian randomization analysis and effects of weight change. PLoS Med. 11, e1001765, https://doi.org/10.1371/journal.pmed.1001765 (2014).

15. Soma-Pillay, P., Nelson-Piercy, C., Tolppanen, H. \& Mebazaa, A. Physiological changes in pregnancy. Cardiovasc. J. Afr. 27, 89-94, https://doi.org/10.5830/CVJA-2016-021 (2016).

16. Hellmuth, C. et al. Association of maternal prepregnancy bmi with metabolomic profile across gestation. Int. J. Obes. (Lond.) 41, 159-169, https://doi.org/10.1038/ijo.2016.153 (2017).

17. Alexandre-Gouabau, M. C. et al. Maternal and cord blood lc-hrms metabolomics reveal alterations in energy and polyamine metabolism, and oxidative stress in very-low birth weight infants. J. Proteome Res. 12, 2764-2778, https://doi.org/10.1021/pr400122v (2013).

18. Tea, I. et al. $1 \mathrm{~h}-\mathrm{nmr}$-based metabolic profiling of maternal and umbilical cord blood indicates altered materno-foetal nutrient exchange in preterm infants. PLoS One 7, e29947, https://doi.org/10.1371/journal.pone.0029947 (2012).

19. Horgan, R. P. et al. Metabolic profiling uncovers a phenotypic signature of small for gestational age in early pregnancy. J. Proteome Res. 10, 3660-3673, https://doi.org/10.1021/pr2002897 (2011).

20. Li, J. et al. Maternal pcaac38:6 is associated with preterm birth - a risk factor for early and late adverse outcome of the offspring. Kidney Blood Press. Res. 41, 250-257, https://doi.org/10.1159/000443428 (2016).

21. Hellmuth, C. et al. Maternal metabolomic profile and fetal programming of offspring adiposity: Identification of potentially protective lipid metabolites. Mol. Nutr. Food Res. 63, e1700889, https://doi.org/10.1002/mnfr.201700889 (2019).

22. Diaz, S. O. et al. Metabolic biomarkers of prenatal disorders: An exploratory $\mathrm{nmr}$ metabonomics study of second trimester maternal urine and blood plasma. J. Proteome Res. 10, 3732-3742, https://doi.org/10.1021/pr200352m (2011).

23. Maitre, L. et al. Maternal urinary metabolic signatures of fetal growth and associated clinical and environmental factors in the inma study. BMC Med. 14, 177, https://doi.org/10.1186/s12916-016-0706-3 (2016).

24. Walsh, J. M., Wallace, M., Brennan, L. \& McAuliffe, F. M. Early pregnancy maternal urinary metabolomic profile and later insulin resistance and fetal adiposity. J. Matern. Fetal Neonatal Med. 28, 1697-1700, https://doi.org/10.3109/14767058.2014.966672 (2015).

25. Maitre, L. et al. Urinary metabolic profiles in early pregnancy are associated with preterm birth and fetal growth restriction in the rhea mother-child cohort study. BMC Med. 12, 110, https://doi.org/10.1186/1741-7015-12-110 (2014).

26. Graca, G. et al. Uplc-ms metabolic profiling of second trimester amniotic fluid and maternal urine and comparison with $\mathrm{nmr}$ spectral profiling for the identification of pregnancy disorder biomarkers. Mol. Biosyst. 8, 1243-1254, https://doi.org/10.1039/ c2mb05424h (2012).

27. Graca, G. et al. Impact of prenatal disorders on the metabolic profile of second trimester amniotic fluid: A nuclear magnetic resonance metabonomic study. J. Proteome Res. 9, 6016-6024, https://doi.org/10.1021/pr100815q (2010).

28. Menon, R. et al. Amniotic fluid metabolomic analysis in spontaneous preterm birth. Reprod. Sci. 21, 791-803, https://doi. org/10.1177/1933719113518987 (2014).

29. Heazell, A. E., Bernatavicius, G., Warrander, L., Brown, M. C. \& Dunn, W. B. A metabolomic approach identifies differences in maternal serum in third trimester pregnancies that end in poor perinatal outcome. Reprod. Sci. 19, 863-875, https://doi. org/10.1177/1933719112438446 (2012).

30. Ciborowski, M. et al. Potential first trimester metabolomic biomarkers of abnormal birth weight in healthy pregnancies. Prenat. Diagn. 34, 870-877, https://doi.org/10.1002/pd.4386 (2014).

31. Kadakia, R. et al. Maternal metabolites during pregnancy are associated with newborn outcomes and hyperinsulinaemia across ancestries. Diabetologia 62, 473-484, https://doi.org/10.1007/s00125-018-4781-1 (2019).

32. Sandler, V. et al. Associations of maternal bmi and insulin resistance with the maternal metabolome and newborn outcomes. Diabetologia 60, 518-530, https://doi.org/10.1007/s00125-016-4182-2 (2017).

33. Thomas, M. M. et al. Metabolite profile of cervicovaginal fluids from early pregnancy is not predictive of spontaneous preterm birth. Int. J. Mol. Sci. 16, 27741-27748, https://doi.org/10.3390/ijms161126052 (2015).

34. Ghartey, J., Bastek, J. A., Brown, A. G., Anglim, L. \& Elovitz, M. A. Women with preterm birth have a distinct cervicovaginal metabolome. Am. J. Obstet. Gynecol. 212, 776 e771-776 e712, https://doi.org/10.1016/j.ajog.2015.03.052 (2015).

35. Auray-Blais, C., Raiche, E., Gagnon, R., Berthiaume, M. \& Pasquier, J.-C. Metabolomics and preterm birth: What biomarkers in cervicovaginal secretions are predictive of high-risk pregnant women? Int. J. Mass Spectrom. 307, 33-38, https://doi.org/10.1016/j. ijms.2011.02.009 (2011).

36. Sulek, K. et al. Hair metabolomics: Identification of fetal compromise provides proof of concept for biomarker discovery. Theranostics 4, 953-959, https://doi.org/10.7150/thno.9265 (2014).

37. Kosmides, A. K., Kamisoglu, K., Calvano, S. E., Corbett, S. A. \& Androulakis, I. P. Metabolomic fingerprinting: Challenges and opportunities. Crit. Rev. Biomed. Eng. 41, 205-221 (2013).

38. Soh, S.-E. et al. Cohort profile: Growing up in singapore towards healthy outcomes (gusto) birth cohort study. Int. J. Epidemiol. 43, 1401-1409, https://doi.org/10.1093/ije/dyt125 (2014).

39. Smart, K. F., Aggio, R. B. M., Van Houtte, J. R. \& Villas-Bôas, S. G. Analytical platform for metabolome analysis of microbial cells using methyl chloroformate derivatization followed by gas chromatography-mass spectrometry. Nat. Protoc. 5, 1709, https://doi. org/10.1038/nprot.2010.108 (2010).

40. Stein, S. E. An integrated method for spectrum extraction and compound identification from gas chromatography/mass spectrometry data. J. Am. Soc. Mass Spectrom. 10, 770-781, https://doi.org/10.1016/S1044-0305(99)00047-1 (1999).

41. National institute of standards and technology standard reference database 1 a $v 14$, https://www.nist.gov/srd/nist-standard-referencedatabase-1a-v17 (2014).

42. Aggio, R., Villas-Boas, S. G. \& Ruggiero, K. Metab: An r package for high-throughput analysis of metabolomics data generated by gc-ms. Bioinformatics 27, 2316-2318, https://doi.org/10.1093/bioinformatics/btr379 (2011). 
43. van der Kloet, F. M., Bobeldijk, I., Verheij, E. R. \& Jellema, R. H. Analytical error reduction using single point calibration for accurate and precise metabolomic phenotyping. J. Proteome Res. 8, 5132-5141, https://doi.org/10.1021/pr900499r (2009).

44. Xia, J., Psychogios, N., Young, N. \& Wishart, D. S. Metaboanalyst: A web server for metabolomic data analysis and interpretation. Nucleic Acids Res. 37, W652-W660, https://doi.org/10.1093/nar/gkp356 (2009).

45. Metz, T. O. Metabolic profiling. (Springer, 2011).

46. Sumner, L. W. et al. Proposed minimum reporting standards for chemical analysis. Metabolomics 3, 211-221, https://doi. org/10.1007/s11306-007-0082-2 (2007).

47. Mikolajczyk, R. T. et al. A global reference for fetal-weight and birthweight percentiles. Lancet 377, 1855-1861, https://doi. org/10.1016/s0140-6736(11)60364-4 (2011).

48. Chia, A.-R. et al. A vegetable, fruit, and white rice dietary pattern during pregnancy is associated with a lower risk of preterm birth and larger birth size in a multiethnic asian cohort: The growing up in singapore towards healthy outcomes (gusto) cohort study. Am. J. Clin. Nutr. 104, 1416-1423, https://doi.org/10.3945/ajen.116.133892 (2016).

49. Chen, L. W. et al. Which anthropometric measures best reflect neonatal adiposity? Int. J. Obes. (Lond.) 42, 501-506, https://doi. org/10.1038/ijo.2017.250 (2018).

50. Hamilton, C. M. et al. The phenx toolkit: Get the most from your measures. Am. J. Epidemiol. 174, 253-260, https://doi.org/10.1093/ aje/kwr193 (2011).

51. Appropriate body-mass index for asian populations and its implications for policy and intervention strategies. Lancet 363, 157-163, https://doi.org/10.1016/s0140-6736(03)15268-3 (2004).

52. Alberti, K. G. M. M. \& Zimmet, P. f. Definition, diagnosis and classification of diabetes mellitus and its complications. Part 1: Diagnosis and classification of diabetes mellitus. Provisional report of a who consultation. Diabet. Med. 15, 539-553 (1998).

53. Chia, A.-R. et al. Adherence to a healthy eating index for pregnant women is associated with lower neonatal adiposity in a multiethnic asian cohort: The growing up in singapore towards healthy outcomes (gusto) study. Am. J. Clin. Nutr. 107, 71-79, https://doi.org/10.1093/ajcn/nqx003 (2018).

54. Padmapriya, N. et al. Physical activity and sedentary behavior patterns before and during pregnancy in a multi-ethnic sample of asian women in singapore. Matern Child Health J 19, 2523-2535, https://doi.org/10.1007/s10995-015-1773-3 (2015).

55. Ng, S. et al. High maternal circulating cotinine during pregnancy is associated with persistently shorter stature from birth to five years in an asian cohort. Nicotine Tob. Res., ntyl48-nty148, https://doi.org/10.1093/ntr/nty148 (2018).

56. McDonald, J. H. Handbook of biological statistics. Vol. 2 (sparky house publishing Baltimore, MD, 2009).

57. Benjamini, Y. \& Hochberg, Y. Controlling the false discovery rate: A practical and powerful approach to multiple testing. J R Stat Soc Series B Stat Methodol 57, 289-300 (1995).

58. Chen, L.-W. et al. Maternal macronutrient intake during pregnancy is associated with neonatal abdominal adiposity: The growing up in singapore towards healthy outcomes (gusto) study. J. Nutr. 146, 1571-1579, https://doi.org/10.3945/jn.116.230730 (2016).

59. Schmidt, J. A. et al. Plasma concentrations and intakes of amino acids in male meat-eaters, fish-eaters, vegetarians and vegans: A cross-sectional analysis in the epic-oxford cohort. Eur. J. Clin. Nutr. 70, 306-312, https://doi.org/10.1038/ejcn.2015.144 (2016).

60. Uauy, R., Suri, D. J., Ghosh, S., Kurpad, A. \& Rosenberg, I. H. Low circulating amino acids and protein quality: An interesting piece in the puzzle of early childhood stunting. EBioMedicine 8, 28-29, https://doi.org/10.1016/j.ebiom.2016.05.026 (2016).

61. Gall, W. E. et al. Alpha-hydroxybutyrate is an early biomarker of insulin resistance and glucose intolerance in a nondiabetic population. PLoS One 5, e10883, https://doi.org/10.1371/journal.pone.0010883 (2010).

62. Logan, K. M., Gale, C., Hyde, M. J., Santhakumaran, S. \& Modi, N. Diabetes in pregnancy and infant adiposity: Systematic review and meta-analysis. Arch. Dis. Child. Fetal Neonatal Ed. 102, F65-F72, https://doi.org/10.1136/archdischild-2015-309750 (2017).

63. Aris, I. M. et al. Effect of maternal glycemia on neonatal adiposity in a multiethnic asian birth cohort. J. Clin. Endocrinol. Metab. 99, 240-247, https://doi.org/10.1210/jc.2013-2738 (2014).

64. Karpe, F., Dickmann, J. R. \& Frayn, K. N. Fatty acids, obesity, and insulin resistance: Time for a reevaluation. Diabetes 60, 2441-2449, https://doi.org/10.2337/db11-0425 (2011).

65. Herrera, E. \& Ortega-Senovilla, H. Maternal lipid metabolism during normal pregnancy and its implications to fetal development. Clin. Lipidol. 5, 899-911, https://doi.org/10.2217/clp.10.64 (2010).

66. Montgomery, M. K. et al. Contrasting metabolic effects of medium- versus long-chain fatty acids in skeletal muscle. J. Lipid Res. 54, 3322-3333, https://doi.org/10.1194/jlr.M040451 (2013).

67. Ran-Ressler, R. R., Bae, S., Lawrence, P., Wang, D. H. \& Thomas Brenna, J. Branched-chain fatty acid content of foods and estimated intake in the USA. Br. J. Nutr. 112, 565-572, https://doi.org/10.1017/S0007114514001081 (2014).

68. Su, X. et al. Adipose tissue monomethyl branched-chain fatty acids and insulin sensitivity: Effects of obesity and weight loss. Obesity (Silver Spring) 23, 329-334, https://doi.org/10.1002/oby.20923 (2015)

69. Mika, A. et al. A comprehensive study of serum odd- and branched-chain fatty acids in patients with excess weight. Obesity (Silver Spring) 24, 1669-1676, https://doi.org/10.1002/oby.21560 (2016).

70. Li, M. et al. Symbiotic gut microbes modulate human metabolic phenotypes. Proc. Natl. Acad. Sci. USA 105, 2117-2122, https://doi. org/10.1073/pnas.0712038105 (2008).

71. Phillips, S., Palmer, R. B. \& Brody, A. Epidemiology, toxicokinetics, and health effects of methyl tert-butyl ether (mtbe). J. Med. Toxicol. 4, 115-126, https://doi.org/10.1007/bf03160966 (2008).

72. Calvani, R. et al. Gut microbiome-derived metabolites characterize a peculiar obese urinary metabotype. Int. J. Obes. (Lond.) 34, 1095-1098, https://doi.org/10.1038/ijo.2010.44 (2010).

73. Elliott, P. et al. Urinary metabolic signatures of human adiposity. Sci. Transl. Med. 7, 285ra262, https://doi.org/10.1126/scitranslmed. aaa5680 (2015).

74. Li, X. et al. Comprehensive two-dimensional gas chromatography/time-of-flight mass spectrometry for metabonomics: Biomarker discovery for diabetes mellitus. Anal. Chim. Acta 633, 257-262, https://doi.org/10.1016/j.aca.2008.11.058 (2009).

75. Perng, W. et al. Associations of cord blood metabolites with perinatal characteristics, newborn anthropometry, and cord blood hormones in project viva. Metabolism 76, 11-22, https://doi.org/10.1016/j.metabol.2017.07.001 (2017).

76. Khoo, C. M. et al. Ethnicity modifies the relationships of insulin resistance, inflammation, and adiponectin with obesity in a multiethnic asian population. Diabetes Care 34, 1120-1126, https://doi.org/10.2337/dc10-2097 (2011).

77. Takashina, C. et al. Associations among the plasma amino acid profile, obesity, and glucose metabolism in japanese adults with normal glucose tolerance. Nutr. Metab. (Lond.) 13, 5, https://doi.org/10.1186/s12986-015-0059-5 (2016),

78. Moran-Ramos, S. et al. An amino acid signature associated with obesity predicts 2-year risk of hypertriglyceridemia in school-age children. Sci. Rep. 7, 5607, https://doi.org/10.1038/s41598-017-05765-4 (2017).

79. Geidenstam, N. et al. Amino acid signatures to evaluate the beneficial effects of weight loss. Int. J. Endocrinol. 2017, 12, https://doi. org/10.1155/2017/6490473 (2017)

\section{Acknowledgements}

All authors thank the GUSTO study group, GUSTO study team, Department of Diagnostic and Interventional Imaging, KKH, Department of Diagnostic Imaging, NUH and the study participants; parents and their neonates for their valuable contribution in this study. The GUSTO study group includes Allan Sheppard, Amutha Chinnadurai, Anne Eng Neo Goh, Anne Rifkin-Graboi, Anqi Qiu, Arijit Biswas, Bee Wah Lee, Birit 
F.P. Broekman, Boon Long Quah, Borys Shuter, Chai Kiat Chng, Cheryl Ngo, Choon Looi Bong, Christiani Jeyakumar Henry, Cornelia Yin Ing Chee, Yam Thiam Daniel Goh, Doris Fok, Fabian Yap, George Seow Heong Yeo, Helen Chen, Hugo P S van Bever, Iliana Magiati, Inez Bik Yun Wong, Ivy Yee-Man Lau, Jeevesh Kapur, Jenny L. Richmond, Jerry Kok Yen Chan, Joanna D. Holbrook, Joshua J. Gooley, Keith M. Godfrey, Kenneth Kwek, Kok Hian Tan, Krishnamoorthy Niduvaje, Leher Singh, Lin Lin Su, Lourdes Mary Daniel, Lynette PeiChi Shek, Marielle V. Fortier, Mark Hanson, Mary Foong-Fong Chong, Mary Rauff, Mei Chien Chua, Michael Meaney, Mya Thway Tint, Neerja Karnani, Ngee Lek, Oon Hoe Teoh, P. C. Wong, Peter D. Gluckman, Pratibha Agarwal, Rob M. van Dam, Salome A. Rebello, Seang-Mei Saw, Shang Chee Chong, Shirong Cai, Shu-E Soh, Sok Bee Lim, Chin-Ying Stephen Hsu, Victor Samuel Rajadurai, Walter Stunkel, Wee Meng Han, Wei Wei Pang, Yap-Seng Chong, Yin Bun Cheung, Yiong Huak Chan and Yung Seng Lee. This research is supported by the Singapore Institute for Clinical Sciences, Agency for Science Technology and Research (A*STAR), Singapore; and the Ministry of Business, Innovation, and Employment (MBIE), New Zealand, through the International Relationships Fund. GUSTO is financially supported under Translational Clinical Research (TCR) Flagship Programme on Developmental Pathways to Metabolic Disease (NMRC/TCR/004-NUS/2008; NMRC/TCR/012NUHS/2014) funded by the National Research Foundation (NRF) and administered by the National Medical Research Council (NMRC), Singapore. Additional funding is provided by the Singapore Institute for Clinical Sciences, A*STAR, Singapore. K.M.G. is supported by the National Institute for Health Research through the NIHR Southampton Biomedical Research Centre and the European Union's Erasmus + Capacity-building ENeA $^{\text {SEA }}$ Project and Seventh Framework Programme (FP7/2007-2013), projects EarlyNutrition and ODIN (grant numbers 289346 and 613977). Study sponsors were not involved in the design of the study, statistical analysis and results interpretation.

\title{
Author contributions
}

M.F.-F.C., P.N.B. and N.K. designed the research; K.M.G., F.Y., K.H.T., L.P.-C.S. and Y.S.L. designed and led the GUSTO study; A-R.C. and J.V.d.S. performed the experiment and processed the metabolites data under the guidance of G.W., K.S., T.-L.H., and E.J.M.; I.M.A. contributed to birth data collection, cleaning, and analysis. A-R.C. performed the statistical analysis and drafted the manuscript. M.F.-F.C. and M.S.K. provided statistical input. All authors reviewed the manuscript for important intellectual content.

\section{Competing interests}

L.P.-C.S. and K.M.G. have received reimbursement for speaking at conferences sponsored by companies selling nutritional products. They are part of an academic consortium that has received research funding from Abbott Nutrition, Nestle, and Danone. The other authors have no potential conflicts of interest to disclose.

\section{Additional information}

Supplementary information is available for this paper at https://doi.org/10.1038/s41598-020-66026-5.

Correspondence and requests for materials should be addressed to A.-R.C. or M.F.-F.C.

Reprints and permissions information is available at www.nature.com/reprints.

Publisher's note Springer Nature remains neutral with regard to jurisdictional claims in published maps and institutional affiliations.

\begin{abstract}
(c) (i) Open Access This article is licensed under a Creative Commons Attribution 4.0 International License, which permits use, sharing, adaptation, distribution and reproduction in any medium or format, as long as you give appropriate credit to the original author(s) and the source, provide a link to the Creative Commons license, and indicate if changes were made. The images or other third party material in this article are included in the article's Creative Commons license, unless indicated otherwise in a credit line to the material. If material is not included in the article's Creative Commons license and your intended use is not permitted by statutory regulation or exceeds the permitted use, you will need to obtain permission directly from the copyright holder. To view a copy of this license, visit http://creativecommons.org/licenses/by/4.0/.
\end{abstract}

(C) The Author(s) 2020 